\title{
Characterization and modeling of drift noise in Fourier transform spectroscopy: implications for signal processing and detection limits
}

\author{
Geoff Hazel, Frank Bucholtz, and Ishwar D. Aggarwal
}

\begin{abstract}
A theoretical analysis of long-term drift noise in Fourier transform spectroscopy is presented. Theoretical predictions are confirmed by experiment. Fractional Brownian motion is employed as a stochastic process model for drift noise. A formulation of minimum detectable signal is given that properly accounts for drift noise. The spectral exponent of the low-frequency drift noise is calculated from experimental data. A frequency-dependent optimal spectrum averaging time is found to exist beyond which the minimum detectable signal increases indefinitely. It is also shown that the minimum detectable signal in an absorbance or transmission measurement degrades indefinitely with the time elapsed since background spectrum acquisition. () 1997 Optical Society of America

Key words: Fourier transform spectroscopy, flicker noise, fractional Brownian motion, mid-IR Spectroscopy, drift noise.
\end{abstract}

\section{Introduction}

Fourier transform (FT) spectroscopy exhibits longterm drift (or flicker) noise. ${ }^{1}$ Noise of this type, also known as $1 / f$ noise, is characterized by a lowfrequency noise spectrum with $1 / f^{\alpha}$ frequency dependence. It is ubiquitous in nature and has been studied in detail in the contexts of the stability of atomic frequency standards and the statistics of nuclear beta decay. ${ }^{2,3}$ The presence of this noise in the FT spectrometer presents several problems for the spectroscopist, including the loss of measurement precision with time elapsed since background spectrum acquisition (background spectrum age), the failure of the $1 / \sqrt{T}$ rule for noise reduction for time- $T$ averaging, and the loss of long-term calibration stability. The importance of these problems justifies a careful theoretical and experimental investigation of drift noise in the FT spectrometer, which this study provides. Although the analysis presented here concentrates on the FT spectrometer, the theoretical de-

The authors are with the U.S. Naval Research Laboratory, Code 5606, Washington, D.C. 20375. The permanent address of G. Hazel is the Fiber and Electro-Optics Research Center, Virginia Polytechnic Institute, Blacksburg, Virginia 24062-0356.

Received 30 January 1997; revised manuscript received 30 May 1997.

0003-6935/97/276751-09\$10.00/0

(C) 1997 Optical Society of America velopment and modeling methods are applicable to all interferometric sensors subject to long-term drift noise.

First we present a mathematical representation of the FT spectrometer measurement that is conducive to noise analysis. Next, two random process models, fractional Brownian motion (fBm) and noisy fractional Brownian motion ( $\mathrm{nfBm})$, are proposed for modeling spectrometer drift noise. Some properties of $\mathrm{fBm}$ and $\mathrm{nfBm}$ are developed. A formulation of minimum detectable signal is developed that includes the influence of drift noise. Some implications for data processing and for the definition of detection limits are discussed next. Finally a description and analysis of experimental observations of spectrometer drift noise are given. Although the experimental observations described are of mid-IR absorption, the formalism developed also applies to emission spectroscopy since the formalism makes no assumptions about the physical sources of the noise.

One can formulate the question of minimum detectable signal, or detection limit, in the FT spectrometer as follows: a background spectrum, consisting of scans averaged over $T$ seconds is collected. Then $P$ seconds after the background collection began, a sample spectrum is collected also consisting of scans averaged over $T$ seconds. The sample spectrum is compared with the background spectrum and a decision is made as to the presence of a chemical analyte. If the sample spectrum differs from the background 
by more than a threshold, $x$, the analyte is judged to be present, otherwise it is judged absent. Associated with a choice of threshold is a probability of error. The detection limit is then defined as the smallest threshold for which the probability of error is smaller than some desired confidence level.

In this paper we investigate the dependence of the detection limit defined in this way on averaging time $T$ and measurement period $P$. The results show the existence of an optimal averaging time beyond which additional averaging causes the detection limit to degrade without bound. It is found that this optimal averaging time is strongly dependent on spectral frequency. In addition, the importance of the use of recent background spectra is confirmed. The spectral exponent, $\alpha$, of the drift-noise power spectral density is measured as a function of IR frequency and is found to lie between 2.5 and 3 . Some proposals are given for data processing techniques that optimize the signal-to-noise ratio in the presence of drift noise.

\section{Representing the Fourier Transform Measurement}

\section{A. Stationary Ergodic Case}

Suppose that $v(t)$ is the analytic signal representation of the optical field of the infrared source, where $t$ is time. The signal incident on the photodetector at the output of a FT interferometer is $v(t)+v(t+\theta)$, where $\theta$ is the interferometer path delay. The interference term measured at the output of the photodetector is then just the cross term $v^{*}(t) v(t+\theta)$, where the asterisk denotes the complex conjugate.

If $v(t)$ is assumed to be a wide-sense stationary ergodic stochastic process, then the interferogram, $\gamma(\theta)$, measured by the spectrometer can be represented by the expectation $(\mathbf{E})$ of $v(t)$ :

$$
\gamma(\theta)=\mathbf{E}\left[v^{*}(t) v(t+\theta)\right]
$$

which is just the autocorrelation function of the random process. Note that the assumption of stationarity implies that $\gamma$ is not a function of $t$. The FT measurement is completed by computing the Fourier transform, $S(\omega)$, of the measured interferogram:

$$
S(\omega)=\int_{-\infty}^{\infty} \gamma(\theta) \exp (-j \omega \theta) \mathrm{d} \theta,
$$

which, by definition, is the power spectral density of $v(t)$.

The difficulty with Eq. (2) from the standpoint of noise analysis is that it is not a function of time. In practice, estimates of $\gamma$ are constructed over finite measurement times, and sequences of such estimates are observed to fluctuate in time. These fluctuations are caused by both changes in the spectral content of the radiation as well as noise in the detection system. Furthermore, in practice, the assumptions of stationarity and ergodicity may not be justified. To analyze these fluctuations a more sophisticated representation of the FT measurement is needed.
B. Representing the Time-Varying Spectrum Estimate

To represent the FT measurement more accurately we must explicitly consider the finite duration of the measurement. Hence, if the output of the photodetector is assumed to be the average of the incident optical field over a time window of length $W$, the interferogram estimate can be expressed as

$$
\hat{\gamma}(t, \theta)=\frac{1}{W} \int_{t-W / 2}^{t+W / 2} v^{*}(\tau) v(\tau+\theta) \mathrm{d} \tau .
$$

It is convenient to treat the optical field as a composition of an underlying stochastic process that is stationary and ergodic with one or more noise processes that may or may not be stationary. For example, one might wish to analyze a thermal radiation source governed by the Planck blackbody law, but with $1 / f$ temperature noise.

To this end, consider the case in which $v(t)$ is a composition of a high-frequency optical field signal $s(t ; \eta)$, and a low-frequency noise process $n(t)$, where $\eta$ is a random parameter to $s(t ; \eta)$. For a given value of the parameter $\eta, s(t ; \eta)$ is assumed to be wide-sense stationary and mean-square ergodic with power spectral density, $S_{s}(\omega ; \eta)$. In particular, it is assumed that time averages over $s(t ; \eta)$ with $\eta$ held constant converge in mean square to the corresponding conditional expectations of $s(t ; \eta)$ given $\eta$. The noise process $n(t)$ can be nonstationary and nonergodic. The specific form of the composition is then $v(t)=s[t$; $n(t)]$.

The following two additional assumptions are made regarding the length of the detector averaging time $W$ compared to the time scales of fluctuations of $s(t ; \eta)$ and $n(t)$ :

(1) $W$ is small compared to the time scale of fluctuations of $n(t)$, and

(2) $W$ is large compared to the time scale of fluctuations of $s(t ; \eta)$.

This allows us to develop Eq. (3) as follows:

$$
\begin{aligned}
\hat{\gamma}(t, \theta)= & \frac{1}{W} \int_{t-W / 2}^{t+W / 2} s^{*}[\tau ; n(\tau)] s[\tau+\theta ; n(\tau+\theta)] \mathrm{d} \tau \\
\approx & \frac{1}{W} \int_{t-W / 2}^{t+W / 2} s^{*}[\tau ; n(\tau)] s[\tau+\theta ; n(t+\theta)] \mathrm{d} \tau \\
\approx & \lim _{W \rightarrow \infty} \frac{1}{W} \int_{t-W / 2}^{t+W / 2} s^{*}[\tau ; n(t)] s[\tau+\theta ; n(t+\theta)] \mathrm{d} \tau \\
= & \mathbf{E}\left\{\left(s^{*}[\tau ; n(\tau)] \mid\{n(\tau)=n(t), \forall \tau\}\right)\right. \\
& \times(s[\tau+\theta ; n(\tau+\theta)] \mid\{n(\tau+\theta) \\
= & n(t+\theta), \forall \tau\})\},
\end{aligned}
$$

where the first approximation is due to assumption number 1 , above, the second approximation is due to assumption number 2 , and the last equality is due to 
the assumed ergodicity of $s(t ; \eta)$. The limit is understood in the mean-square sense. Note that the expectation in the last line above is the correlation between two instances of the process $s(t ; \eta)$ conditioned on possibly different values of its parameter $\eta$. However, in the FT spectrometer, and in many other interferometric systems with sources of finite coherence, the interferometer path delay $\theta$ remains within a range that is small compared to the time scale of fluctuations of the noise process $n(t)$. Hence we can make the additional approximation that $n(t+\theta) \approx$ $n(t)$, which allows

$$
\begin{aligned}
\hat{\gamma}(t, \theta) \approx & \mathbf{E}\left\{\left(s^{*}[\tau ; n(\tau)] \mid\{n(\tau)=n(t), \forall \tau\}\right)\right. \\
& \times(s[\tau+\theta ; n(\tau)] \mid\{n(\tau) \\
= & n(t), \forall \tau\})\} \\
\equiv & \gamma_{s \mid n(t)}(\theta)
\end{aligned}
$$

where we have defined the conditional autocorrelation function of $s(t)$ given a time-varying parameter $n(t)$, and we have dropped the dependence on $\tau$ because of the assumed conditional stationarity of $s(t)$.

To complete the analysis, we carry out the FT of the measurement with respect to $\theta$ to obtain

$$
\hat{S}(\omega)=\mathscr{F}_{\theta}\{\hat{\gamma}(t, \theta)\} \approx S_{s \mid n(t)}(\omega),
$$

which is the conditional power spectral density of $s(t)$ given a time-varying parameter $n(t)$. Note that for each value of $\omega, S_{s \mid n(t)}(\omega)$ is a stochastic process evolving in time, which is the stochastic process that satisfies the intuitive notion of an underlying stationary process governed by a power spectral density that is subject to slowly varying drift noise. It is well known that in an FT spectrometer the noise can vary across $\omega$, which is allowed by Eq. (6). For example, there may be drift fluctuations in the strength of a particular absorption feature. It has been shown experimentally ${ }^{4}$ and theoretically, ${ }^{1}$ and will be observed again in Section 6 that such fluctuations remain localized in the FT spectrum. For this reason, in Section 6 we treat the noise at each $\omega$ separately. Before proceeding further with the analysis we must identify a random process model for the drift noise that captures the important features of observed noise processes.

\section{Modeling Drift Noise with Fractional Brownian Motion}

Fractional Brownian motion $(\mathrm{fBm})^{5,6}$ is a random process model that is useful for representing drift noise and other noises that have divergent power spectral densities with $1 / f^{\alpha}$ low-frequency behavior. It is a nonstationary random process that nonetheless possesses a meaningful power spectral density ${ }^{7}$ and that contains classical Brownian motion as a special case.

\section{A. Fractional Brownian Motion}

\section{Definition}

A $\mathrm{fBm}$ process $f(t)$ is formally defined as a zero-mean Gaussian process with $f(t)=0$ for $t \leq 0$ with probability one and with autocovariance

$$
\gamma_{f}\left(t_{1}, t_{2}\right)=\frac{c_{\gamma}}{2}\left(\left|t_{1}\right|^{\alpha-1}+\left|t_{2}\right|^{\alpha-1}+\left|t_{1}-t_{2}\right|^{\alpha-1}\right),
$$

where the parameter $\alpha \in(1,3)$ and the constant $c_{\gamma} \in$ $[0, \infty)$ is arbitrary. Note that this implies that the variance of $f(t)$ grows in time as $t^{\alpha-1}$.

\section{Properties}

Although $f(t)$ is nonstationary it does have a power spectral density given by ${ }^{7}$

$$
S_{f}(\omega)=\frac{c_{S}}{\omega^{\alpha}},
$$

where $c_{S}=4 c_{\gamma}(\alpha-1) \Gamma(\alpha-1) \sin [\pi / 2(\alpha-1)]$. Hence the parameter $\alpha$ is the spectral exponent of the process. The differences in $f(t)$ over a time $T$ are stationary Gaussian random variables with zero mean and variance $c_{\gamma} T^{\alpha-1}$. Hence ${ }^{6}$

$$
\begin{aligned}
\mathbf{P}[f(t+T)-f(t) & \leq x] \\
& =\frac{1}{\left(2 \pi c_{\gamma} T^{\alpha-1}\right)^{1 / 2}} \int_{-\infty}^{x} \exp \left(-\frac{u^{2}}{2 c_{\gamma} T^{\alpha-1}}\right) \mathrm{d} u
\end{aligned}
$$

is the probability that the process will change by no more than $x$ in time $T$.

\section{Allan Variance}

The Allan variance of a random process ${ }^{8,9}$ is a measure of the variability in consecutive finite-length estimates of the process mean. It is defined as the variance of the difference between consecutive finitelength sample averages. That is,

$$
V(T)=\frac{1}{2 T^{2}} \mathbf{E}\left\{\int_{t-T}^{t}[f(\tau)-f(\tau-T)] \mathrm{d} \tau\right\}^{2} .
$$

The Allan variance can be related to the power spectral density through a transform theorem given by Van Vliet and Handel. ${ }^{10}$ It can be shown ${ }^{7,10}$ that the Allan variance of $\mathrm{fBm}$ is $c_{V} T^{\alpha-1}$, where

$$
c_{V}=\frac{4 c_{\gamma}\left(2^{\alpha-1}-1\right)}{\alpha(\alpha+1)} \text {. }
$$

\section{Parameter Estimation}

Parameter estimation for $\mathrm{fBm}$ requires some care. The sample average, for example, is an efficient estimator for the process mean. however its rate of convergence is slow. ${ }^{11}$ Hence the sample average of a finite data record may not be a good estimate of the process mean. The sample variance is even less useful. Since the process variance grows in time [Eq. 
(7)] the sample variance is clearly not a good estimate of the process variance. Neither is the sample variance a good estimator of the constant $c_{\gamma} \cdot{ }^{11}$ However, the Allan variance can be efficiently estimated from finite data records, as can the spectral exponent $\alpha$. The Allan variance can be estimated by its corresponding sample variance, which is a sample variance of the stationary increment process. Leu and Papamarcou ${ }^{12}$ discuss three techniques for estimating $\alpha$. They recommend the estimation of $\alpha$ from the slope of the log-log plot of the Allan variance versus averaging time $(V(T))$, which is the method employed here.

\section{Noisy Fraction Brownian Motion}

Guillemet et al. discussed a process that they denote noisy $\mathrm{fBm}(\mathrm{nfBm})$, which is simply the sum of $\mathrm{fBm}$ with a zero-mean white Gaussian process of variance $\sigma^{2}{ }^{13}$ This combined process proved useful for our study. One can easily show that this process has lag-T increments that are zero-mean, stationary Gaussian random variables with variance $2 \sigma^{2}+$ $c_{\gamma} T^{\alpha-1}$. Hence Eq. (9) can be easily extended to this case. Further, the Allan variance of $\mathrm{nfBm}$ is given by

$$
V_{n f B m}(T)=\frac{\sigma^{2}}{T}+c_{V} T^{\alpha-1} .
$$

Equation (12) is interesting because for $\alpha>1$ it has a minimum that is easily seen to occur at

$$
T_{0}=\left[\frac{\sigma^{2}}{c_{V}(\alpha-1)}\right]^{1 / \alpha} \text {. }
$$

\section{Implications for Data Processing and Detection Limits}

The presence of slow drift noise in a FT spectrometer measurement has some important implications for data processing and for the definition of detection limits. In particular, Eq. (12) implies that extended averaging of spectrometer scans does not reduce the noise in the measurement. Indeed, for $\alpha>1 \mathrm{Eq}$. (13) gives an optimal averaging time beyond which the measurement noise begins to grow. Further, the concept of minimum detectable signal in the presence of slow drift noise must be carefully reexamined.

\section{A. Definition of the Detection Limit}

The notion of detection limit formulated in Section 1 can easily be computed. Considering only the probability of a false positive, the probability of error is just the probability that the noise process governing the background spectrum generated the observed sample spectrum. Now if the analyte were absent, the difference between the two measured spectra could be assumed to be a zero-mean Gaussian random variable with variance $\sigma_{d}^{2}$ given by

$$
{\sigma_{d}}^{2}=\frac{1}{2 T^{2}} \mathbf{E}\left[\int_{t}^{t+T} f(\tau) \mathrm{d} \tau-\int_{t}^{t+T} f(\tau-P) \mathrm{d} \tau\right]^{2} .
$$

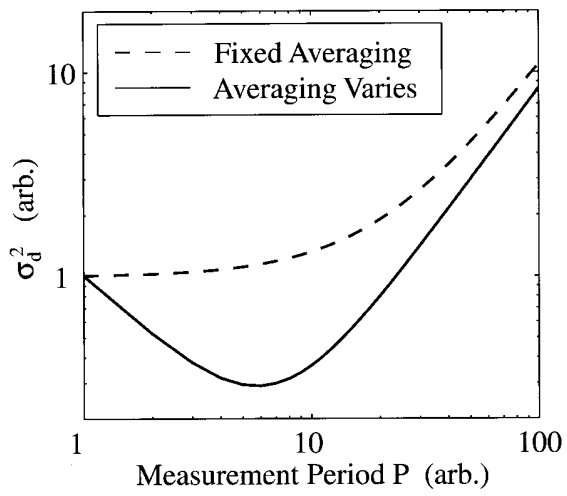

Fig. 1. Plots of theoretical measurement variance $\left(\sigma_{d}^{2}\right)$ for noisy fractional Brownian motion with $\alpha=2.5$ and $c_{\gamma} / \sigma^{2}=0.02$. Dashed curve, averaging time $T$ is fixed and measurement period $P$ varies; in solid curve, averaging time and measurement period vary together with zero measurement delay $(T=P)$.

This is just the variance of the difference between averaged blocks of length $T$ spaced by time $P$. Hence the probability of error can be computed from an expression analogous to Eq. (9). A common choice of the threshold is $x=3 \sigma_{d}$, which leads to a probability of error of 0.0013 .

If we make the usual assumption that $f(t)$ is zeromean Gaussian white noise with variance $\sigma^{2}$, we can easily show that $\sigma_{d}^{2}=\sigma^{2} / T$. This is the standard result, which is that the detection limit is independent of measurement period $P$ and improves with averaging time as $1 / \sqrt{T} .^{14}$

For fBm, however, one can show by a procedure similar to that in Solo ${ }^{7}$ that $\sigma_{d}^{2}$ is given by

$$
\begin{aligned}
\sigma_{d}^{2}= & \frac{c_{V}}{4\left(2^{\alpha-1}-1\right) T^{2}}\left[(P+T)^{\alpha+1}\right. \\
& \left.+(P-T)^{\alpha+1}-2 T^{\alpha+1}-2 P^{\alpha+1}\right],
\end{aligned}
$$

which diverges in both $T$ and $P$.

If additive white Gaussian noise is included, then the result for $\mathrm{nfBm}$ gives

$$
\begin{aligned}
\sigma_{d}^{2}= & \frac{\sigma^{2}}{T}+\frac{c_{V}}{4\left(2^{\alpha-1}-1\right) T^{2}}\left[(P+T)^{\alpha+1}\right. \\
& \left.+(P-T)^{\alpha+1}-2 T^{\alpha+1}-2 P^{\alpha+1}\right] .
\end{aligned}
$$

Equations (15) and (16) reduce to the usual Allan variance in the case $T=P$. In particular, Eq. (16) possesses a minimum just as does Eq. (12).

\section{B. Implications}

\section{Importance of Recent Background Spectra}

The dashed curve in Fig. 1 shows a plot of Eq. (16) for $\alpha=2.5, c_{\gamma} / \sigma^{2}=0.02, T=1$, and $P \in\{1,2, \ldots, 100\}$. Clearly the detection limit degrades without bound as the delay between measurements increases. This result underscores the importance of using recent background spectra for all FT spectrometer measurements. Other approaches to experimental design that attempt to compensate for drift noise also merit 
consideration. Examples can include techniques for simultaneous or nearly simultaneous measurement of background and sample spectra, and normalization of spectra by total detected power. Care should be used in the design of such schemes, however, since they introduce their own sources of drift noise.

\section{Choice of Averaging Times}

The solid curve in Fig. 1 shows a plot of the same equation as a function of averaging time $T$ with no measurement delay $(T=P)$. This clearly shows the existence of an optimal averaging time and agrees with the experimental results discussed in Section 5 . This result suggests that one must exercise some caution in choosing a spectrometer averaging time. While it is well known that the $1 / \sqrt{T}$ noise reduction implied by the white noise assumption does not hold indefinitely, it is perhaps not widely appreciated that extended averaging can actually increase the measurement noise.

In light of this, Eq. (13) seems to provide a clear choice for averaging time $T$. However, as we see in Section 6 the value of this optimal averaging time can vary strongly as a function of spectral frequency. Use of a different averaging time at each spectral frequency turns out not to reduce the noise except at those spectral frequencies for which the optimal averaging time is longest. The reason is that to make the desired calculation the data record must be partitioned into fixed epochs of length equal to the maximum desired averaging time. In computing the average at frequencies for which an averaging length less than the maximum is desired, one must discard the extra data. This does not yield the optimum noise performance. In fact, Eq. (16) indicates that a calculation that discards data in this manner is actually noisier than if it averages over the full, suboptimal, data epoch length. Hence, one should at a minimum choose an averaging time that minimizes the variance over the spectral region of interest.

If a multivariate technique such as principal components analysis (PCA) is to be employed over a wide region of the spectrum with diverse statistics, one must approach the problem differently. One possibility would be to project a large background data set onto the desired PCA axes and compute the Allan variance of the PCA scores as a function of averaging time. An optimal averaging time for one or a combination of principal components could then be found. Another approach that may prove valuable in continuous monitoring situations is to perform nfBmmodel-based maximum likelihood detection with the continuously collected spectra viewed as a random field governed by $\mathrm{nfBm}$.

\section{Experimental Observation of Fourier Transform Infrared Drift Noise}

A set of experiments was performed to acquire FTInfrared (FTIR) spectrometry background data sets suitable for characterization of long-term drift noise.

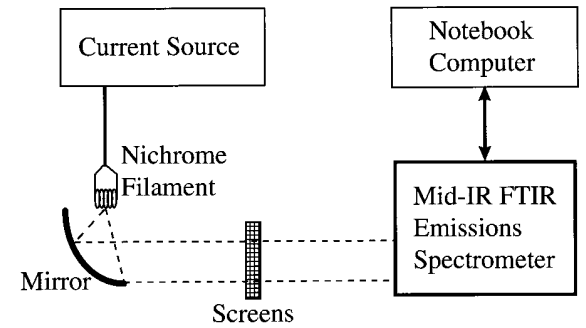

Fig. 2. Experimental Setup. The current source was a Kepco Model BOP 36-6M; the spectrometer was a Midac Model 2406-C portable emission spectrometer.

\section{A. Description of Experiment}

The experiment consisted of continuously collecting mid-IR FT background spectra. Two data sets were collected consecutively. The first set consisted of 2000 spectra each containing four averaged scans. Each of the 2000 spectra required an average of 1.468 $\mathrm{s}$ to collect, for a total data collection time of approximately $50 \mathrm{~min}$. The second data set consisted of 3000 spectra each containing 15 averaged scans. Each of the 3000 spectra required an average of 4.264 $\mathrm{s}$ to collect, for a total collection time of approximately $3 \mathrm{~h}$ and $33 \mathrm{~min}$. The timing of the first data set was chosen to use the maximum rate at which spectra could be collected. The second set was chosen to maximize the total measurement time, which was limited by the hold time of the liquid-nitrogen Dewar that cooled the FTIR photodetector.

Figure 2 is a diagram of the experimental setup. A Kepco model BOP 36-6M power supply provided approximately $3.5 \mathrm{~A}$ at $3 \mathrm{~V}$ dc to a Nichrome filament source. This source is typical of those used in commercial FTIR instruments in the mid-IR. The stability of such sources depends on the stability of the current supply. The current supply used in these experiments was a laboratory-grade stabilized supply. A portion of the radiation from the filament was collimated by a gold-coated parabolic mirror and directed toward the FTIR spectrometer. Aluminum mesh screens were used to attenuate the beam to a suitable power level. The FTIR spectrometer was a Midac Model 2406-C portable emission spectrometer with $\mathrm{ZnSe}$ optics and a liquid-nitrogen-cooled InSb photodetector. Figure 3 shows a typical spectrum in the data sets. The cutoff frequency of the InSb photodetector is approximately $1800 \mathrm{~cm}^{-1}$.

\section{B. Observation of Drift Noise}

Long-term drift noise was readily observed in both experiments. Figures 4 and 5 show the time evolution of the measured FTIR spectra at various spectral frequencies. Figure 4 contains data from the shortterm set and Fig. 5 contains data from the long-term set. The data in both figures were mean centered to facilitate comparison. The presence of both white noise and long-term drift is evident. Furthermore, comparison of the two figures strongly suggests a self-affine process, such as $\mathrm{fBm} .{ }^{5}$ It should be pointed out that, although drift noise is readily ob- 


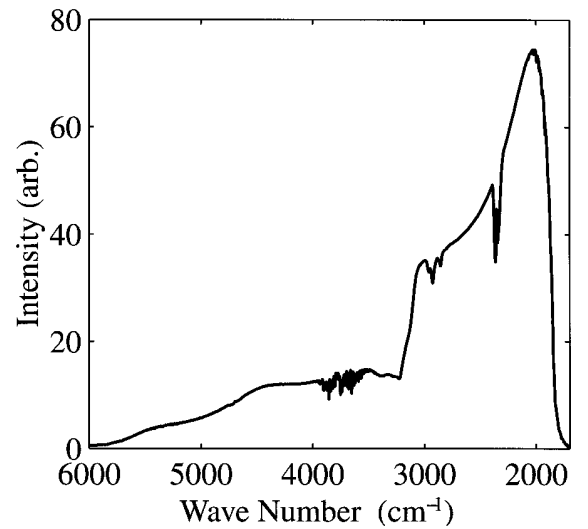

Fig. 3. Typical spectrum from mid-IR FT background data sets. The InSb photodetector cutoff frequency is approximately 1800 $\mathrm{cm}^{-1}$.

served, it is not straightforward to identify the sources of this noise.

Potential sources of drift noise in the system include fluctuations of the source temperature and emissivity, variations in absorption in the beam path, flicker noise in the photodetector and subsequent

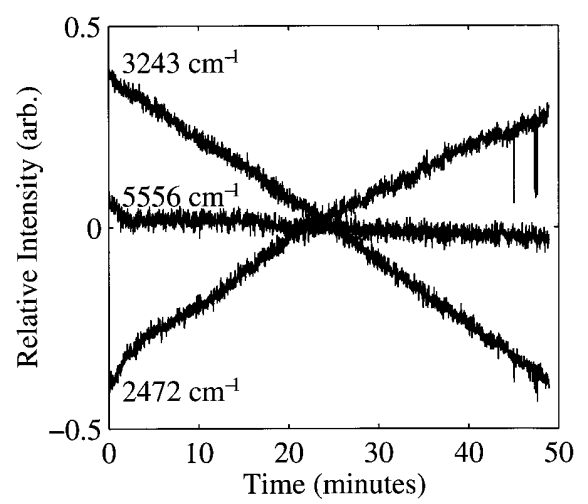

Fig. 4. Time evolution of FTIR spectra at several spectral frequencies from the short-term data set. Data have been mean centered.

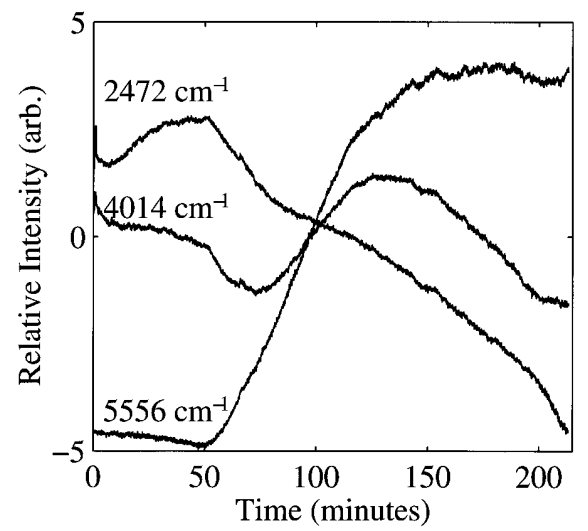

Fig. 5. Time evolution of FTIR spectra at several spectral frequencies from the long-term data set. Data have been mean centered. Note the self-similarity with respect to Fig. 4.

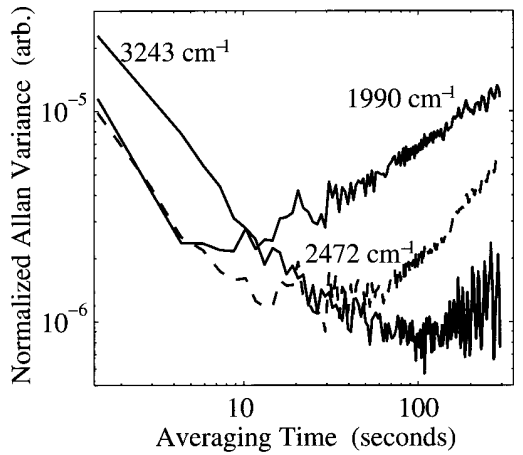

Fig. 6. Allan variance normalized to mean versus averaging time for the short-term data set at three points in the IR spectrum.

electronics, and noise introduced by the numerical data processing. The first two sources, which are evident in the current data, can be reduced in a laboratory instrument with a sealed, purged enclosure. However, the experimental arrangement described here may be more representative of in situ field applications of FT spectroscopy. Additional measurements will be required to attempt to quantify the relative contributions of the various noise sources in the system.

\section{Analysis and Characterization of Experimental Results}

\section{A. Allan Variance versus Averaging Time}

The Allan variance (see Subsection 3.A.3) of both data sets was calculated at each frequency in the FTIR spectrum over a range of averaging times. The short-term data set, for example, consisted of 2000 spectra each including four averaged scans of the spectrometer. First the Allan variance was computed for the 2000 spectra individually, next the 2000 spectra were averaged in consecutive pairs and the Allan variance computed for the resulting 1000 averaged spectra, then the 2000 spectra were averaged over groups of three, and so on. The longest averaging time for the short-term data set consisted of ten groups of 200 averaged spectra. The Allan variance was computed for the long-term data set in the same manner. The longest averaging time for the longterm data set consisted of ten groups of 300 averaged spectra. Figures 6 and 7 show the results for the short- and long-term sets, respectively, at three different points in the mid-IR spectrum.

Examination of these figures confirms the prediction of Eq. (12); namely, that (a) at each point in the spectrum there is an initial decrease of Allan variance with averaging time visible in Fig. 6 that is due to the averaging out of the white noise, and (b) for the large averaging times displayed in Fig. 7, the Allan variance diverges.

It is worth noting that much of the irregularity in the plots in these two figures is due to a computational artifact. When computing $V(T)$ from a data record of fixed length $N$, the data must be partitioned into blocks of length $T$. When $N$ is not a multiple of 


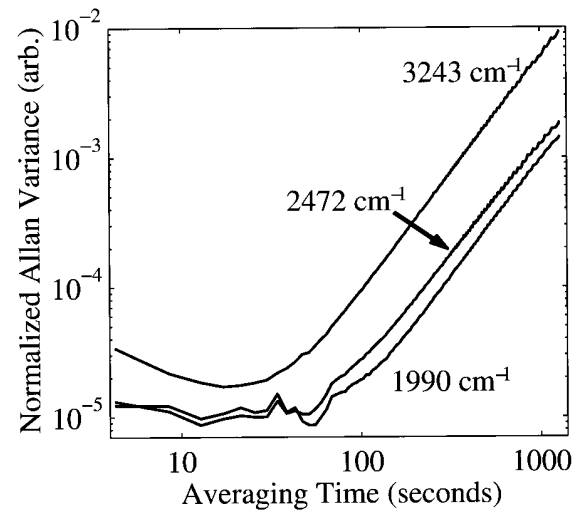

Fig. 7. Allan variance normalized to mean versus averaging time for the long-term data set at three points in the IR spectrum.

$T$, the partition leaves a remainder block of length less than $T$. In the calculation that produced Figs. 6 and 7 the remainder block was included in the Allan variance computation with its contribution scaled in proportion to its length. Nevertheless, the result retains an error ripple that has the shape of the remainder of $N / T$ as a function of $T$. This is particularly visible in the $3243-\mathrm{cm}^{-1}$ trace in Fig. 7 . The effect of this artifact is enhanced when the data record contains sudden jumps, such as those in the $2472-\mathrm{cm}^{-1}$ trace in Fig. 4. However, the artifact does not change the overall shape of the curves and does not influence the conclusions drawn from the data.

\section{B. Optimal Averaging}

The optimal averaging time, that is the averaging time for which the Allan variance was at a minimum and hence the signal-to-noise ratio was at a maximum, was computed for the short-term data set. The result is given in Fig. 8. This plot is ragged due to the artifact discussed in Subsection 6.A. However, it is clear from this figure that the optimal averaging time varies across the spectrum from a minimum of less than $10 \mathrm{~s}$ to a maximum of more than $300 \mathrm{~s}$. Further, it is clear that, if one were

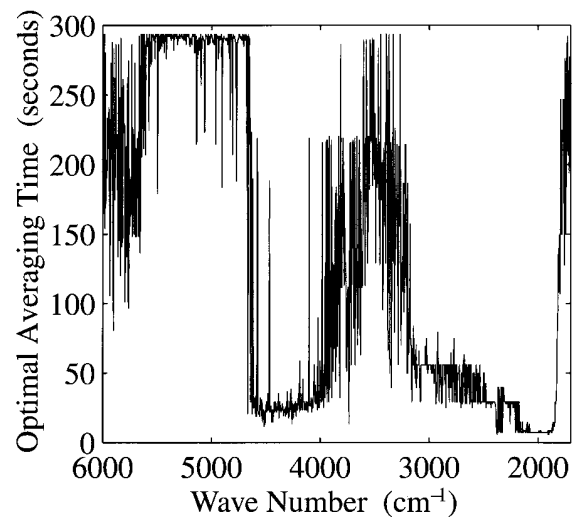

Fig. 8. Averaging time with minimum Allan variance versus spectral frequency for the short-term data set.

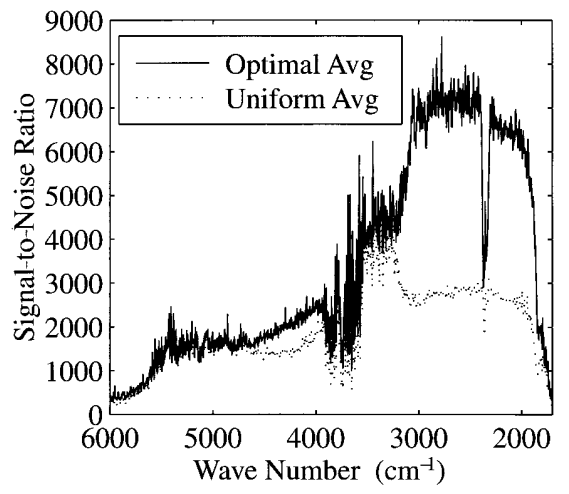

Fig. 9. Signal-to-noise ratio (sample mean divided by square root of Allan variance). For the optimal averaging trace, data were averaged differently at each spectral frequency with the averaging times recommended by Fig. 8. For the uniform averaging trace, data were averaged for $293.6 \mathrm{~s}$ at each spectral frequency. Note that, since such averaging is impossible in practice, the optimal signal-to-noise ratio indicated here cannot be achieved at all spectral frequencies simultaneously.

interested in spectral features that occur, for example, near $3000 \mathrm{~cm}^{-1}$, averaging spectra for more than approximately $1 \mathrm{~min}$ would only increase the measurement noise.

The optimal signal-to-noise ratio obtained by averaging at each spectral frequency with the averaging times shown in Fig. 8 is plotted in Fig. 9. For comparison, the signal-to-noise ratio obtained by averaging for the maximum averaging time of $293.6 \mathrm{~s}$ at each spectral frequency is also plotted. In both cases, the signal-to-noise ratio is computed as the sample mean divided by the square root of the Allan variance. This figure demonstrates that, in spectral regions where drift noise is dominant, particularly between 2000 and $3000 \mathrm{~cm}^{-1}$, averaging in excess of the optimal averaging time degrades the signal-tonoise ratio by more than a factor of 2 . Note that, in practice, as discussed in Subsection 4.B.2, nonuniform averaging cannot be performed without discarding data. Hence, the optimal signal-to-noise ratio indicated in Fig. 9 cannot be achieved at all spectral frequencies simultaneously.

Figure 10 shows an achievable signal-to-noise improvement when 29.36-s averaging, which is nearly optimal between 2000 and $3000 \mathrm{~cm}^{-1}$, is compared with the maximum averaging time of $293.6 \mathrm{~s}$. Note that most of the improvement shown in Fig. 9 is maintained in the regions between 2000 and 3000 $\mathrm{cm}^{-1}$ and between 4000 and $4500 \mathrm{~cm}^{-1}$. However, the signal-to-noise ratio is reduced in regions of the spectrum that are dominated by white noise, such as between 5000 and $6000 \mathrm{~cm}^{-1}$. These results are consistent with Fig. 8.

Figures 9 and 10 demonstrate another feature typical of source flicker noise. The maximally averaged spectrum in each figure is more uniform across spectral frequency than are the optimally averaged spectra. As discussed in Ref. 4, source flicker-noise intensity varies linearly with signal intensity, hence 


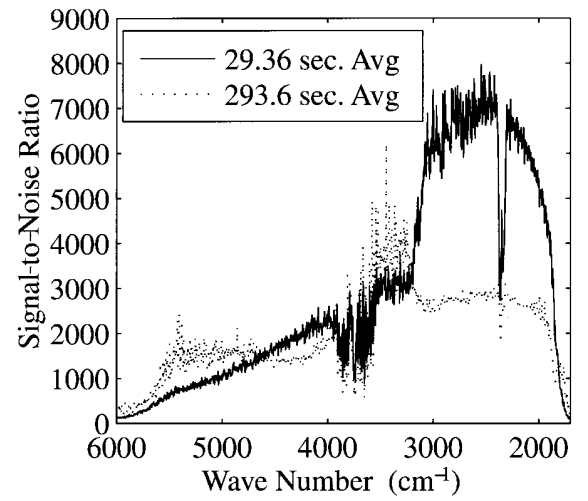

Fig. 10. Signal-to-noise ratio (sample mean divided by square root of Allan variance) for two different averaging times.

leading to a signal-to-noise ratio spectrum that is uniform across spectral frequency. Other noise sources, such as white noise in the detector, tend to be independent of signal intensity and lead to signal-tonoise ratio spectra with shapes similar to the raw spectra. Figures 9 and 10 indicate that excessive averaging enhances signal-dependent noise, such as source flicker noise, leading to more uniform, reduced signal-to-noise ratio spectra. Similar observations of discrete ultraviolet emission lines reported in Ref. 4 allowed detailed conclusions to be drawn regarding sources of observed noise. Such conclusions cannot be drawn from the data described here owing to the lack of discrete lines in the mid-IR and to differences in experimental design. A complete noise characterization involving long-term measurements for each of a variety of experimental configurations is a difficult problem that is beyond the scope of this paper.

\section{Allan Variance Versus Averaging Time for Fixed Data Blocks}

Figures 8 and 9 seem to suggest that one might improve the noise performance of a spectrometer by using different averaging times for each point in the spectrum. For reasons discussed in Subsection 4.B.2, this idea turns out to be false. Figure 11 shows the result of the calculation discussed there for the short-term data set at three different points in

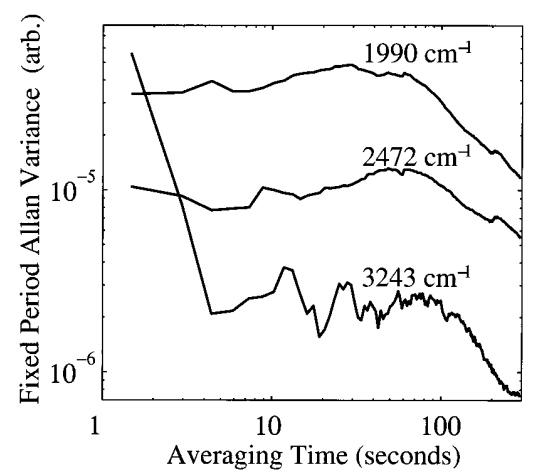

Fig. 11. Allan variance normalized to mean versus averaging time with a fixed measurement period of $P=293.6 \mathrm{~s}$.

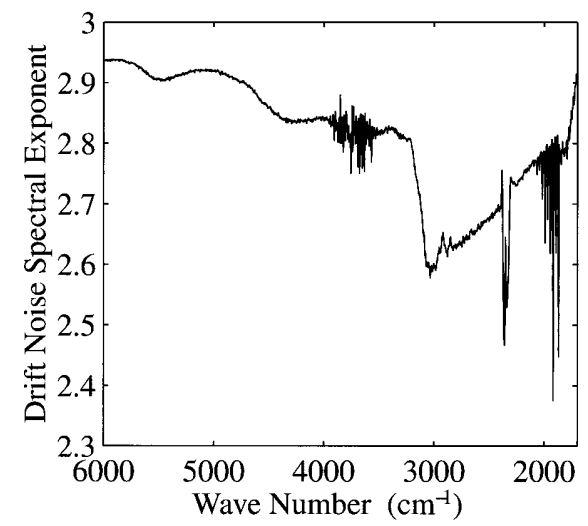

Fig. 12. Low-frequency spectral exponent $(\alpha)$ of drift noise at each IR frequency calculated from the log-log plot of Allan variance versus averaging time.

the spectrum. The calculation uses a fixed measurement period of $P=293.6 \mathrm{~s}$ and varies the averaging time from 1.468 to $293.6 \mathrm{~s}$. This figure confirms that the Allan variance is minimum when no data are discarded.

\section{Drift Noise Spectral Exponent versus Spectral Frequency}

The drift noise spectral exponent [ $\alpha$ in Eq. (8)] was computed from the long-term data set using the loglog plot of the Allan variance as described by Leu and Papamarcou. ${ }^{12}$ Figure 12 shows the result. Several spectral features are visible in this figure, including the $\mathrm{CO}_{2}$ absorption near $2400 \mathrm{~cm}^{-1}$, the $\mathrm{C}-\mathrm{H}$ stretch absorptions near $2850 \mathrm{~cm}^{-1}$, and the water vapor absorption near $3000 \mathrm{~cm}^{-1}$. It is interesting to note that the drift noise in the water and $\mathrm{CO}_{2}$ features is marked by a reduced spectral exponent whereas the noise on the $\mathrm{C}-\mathrm{H}$ stretch feature has a larger spectral exponent than nearby points. One possible explanation is that the $\mathrm{C}-\mathrm{H}$ feature was caused by residual hydrocarbons fixed to optical surfaces in the spectrometer whereas the water and $\mathrm{CO}_{2}$ features were due to vapor in the beam path. If this were the case, one would certainly expect the two features to exhibit qualitatively different statistical behavior.

\section{Conclusions}

A theoretical analysis of FT spectrometer drift noise based on noisy fractional Brownian motion has been presented and compared with experimental observations of spectrometer drift noise. The $1 / f^{\alpha}$ noise spectral exponent was calculated from experimental data and found to vary between 2.5 and 3 across the mid-IR spectrum. Both theory and experiment confirm the existence of an optimal spectrum averaging time for which the minimum detectable signal takes its smallest value. It was observed that, for averaging times $T$ beyond the optimum, the drift noise caused the detection limit to degrade as $T^{\alpha-1}$. Evidence was presented that supports the importance of using recent background spectra in the computation 
of transmission or absorption spectra, and an expression was developed that quantifies the degradation of detection limit with background spectrum age.

It is clear from the above analysis that lowfrequency drift noise is an important factor in the performance of the FT spectrometer. The results presented provide a method of estimating spectrometer detection limits that remains correct in the presence of drift noise. Expressions are provided for predicting the degradation of detection limits with time. Consideration of the results presented here will allow the spectroscopist to achieve the optimum performance of a FT spectrometer in the presence of long-term drift noise.

The authors thank Adrian Papmarcou of the University of Maryland at College Park for helpful discussions regarding fractional Brownian motion.

\section{References}

1. E. Voigtman and J. D. Winefordner, "The multiplex disadvantage and excess low-frequency noise," Appl. Spectrosc. 41(7), 1182-1184 (1987).

2. J. A. Barnes, A. R. Chi, L. S. Cutler, D. J. Healey, D. B. Leeson, T. E. McGunigal, Jr., J. A. Mullen, W. L. Smith, R. L. Sydnor, R. F. C. Vessot, and G. M. R. Winkler, "Characterization of frequency stability," IEEE Trans. Instrum. Meas. IM-20(2), 105-120 (1971).

3. J. Gong, W. H. Ellis, C. M. VanVliet, G. Bosman, and P. Han- del, "Observation of $1 / \mathrm{f}$ noise fluctuations in radioactive decay rates," Trans. Am. Nucl. Soc. 45, 221-222 (1983).

4. S. Marra and G. Horlick, "Signal-to-noise ratio characteristics of an inductively coupled plasma/Fourier transform spectrometer," Appl. Spectrosc. 40, 804-813 (1986).

5. B. B. Mandelbrot and J. W. Van Ness, "Fractional Brownian motions, fractional noises and applications," SIAM Rev. 10(4), 422-437 (1968).

6. K. Falconer, Fractal Geometry: Mathematical Foundations and Applications. (Wiley, Chichester, England, 1990).

7. V. Solo, "Intrinsic random functions and the paradox of $1 / \mathrm{f}$ noise," SIAM J. Appl. Math. 52(1), 270-291 (1992).

8. J. A. Barnes, "Atomic timekeeping and the statistics of precision signal generators," Proc. IEEE 54, 207-220 (1966).

9. D. W. Allan, "Statistics of atomic frequency standards," Proc. IEEE 54(2), 221-230 (1966).

10. C. M. Van Vliet and P. H. Handel, "A new transform theorem for stochastic processes with special application to counting statistics," Physica 113A, 261-276 (1982).

11. J. Beran, "Statistical methods for data with long-range dependence," Stat. Sci. 7(4), 404-427 (1992).

12. J-S. Leu and A. Papamarcou, "On estimating the spectral exponent of fractional Brownian motion," IEEE Trans. Inf. Theory 41(1), 233-244, (1995).

13. H. Guillemet, H. Benali, F. Preteux, and R. DiPaola "Noisy fractional Brownian motion for detection of perturbations in regular textures," in Statistical and Stochastic Methods for Image Processing, E. R. Dougherty, F. Preteux, and J. L. Davidson, eds., Proc. SPIE 2823, 40-51 (1996).

14. M. A. Sharaf, D. L. Illman, and B. R. Kowalski, Chemometrics (Wiley, New York, 1986). 